\title{
Editorial: Cross Adaptation and Cross Tolerance in Human Health and Disease
}

\author{
Ben James Lee ${ }^{1 *}$, Oliver R. Gibson ${ }^{2}$, Charles Douglas Thake ${ }^{3}$, Mike Tipton ${ }^{4}$, \\ John A. Hawley ${ }^{5}$ and James David Cotter 6 \\ ${ }^{1}$ Occupational Performance Research Group, Institute of Sport, University of Chichester, Chichester, United Kingdom, \\ ${ }^{2}$ Department of Life Sciences, Centre for Human Performance, Exercise and Rehabilitation, Brunel University London, \\ Uxbridge, United Kingdom, ${ }^{3}$ Faculty of Health and Life Sciences, Coventry University, Coventry, United Kingdom, ${ }^{4}$ Extreme \\ Environments Laboratory, Department of Sport and Exercise Science, University of Portsmouth, Portsmouth, \\ United Kingdom, ${ }^{5}$ Mary MacKillop Institute for Health Research, Australian Catholic University, Melbourne, VIC, Australia, \\ ${ }^{6}$ School of Physical Education, Sport and Exercise Sciences, University of Otago, Otago, New Zealand
}

Keywords: heat, adaptation, preconditining, hypoxia, nutrition, dehydration

\section{Editorial on the Research Topic}

\section{Cross Adaptation and Cross Tolerance in Human Health and Disease}

Human physiological responses to heat, cold, hypoxia, microgravity, hyperbaria, hypobaria, and fasting are well-studied in isolation. However, in the natural world these stressors are often combined or experienced sequentially (Tipton, 2012). Studies examining human responses to these more realistic, yet relatively complex, circumstances remain sparse, but could provide important insights into an emerging area within human physiology: cross-adaptation (Figure 1) (Lunt et al., 2010; Gibson et al., 2017). Much of the current state of knowledge involves data demonstrating benefits of exercising in hot conditions, prior to performance in hypoxia (Heled et al., 2012; Lee et al., 2014a,b, 2016; Gibson et al., 2015; White et al., 2016; Salgado et al., 2017), with cold to

\section{OPEN ACCESS}

Edited and reviewed by:

Gary Iwamoto,

University of Illinois at

Urbana-Champaign, United States

*Correspondence: Ben James Lee b.lee@chi.ac.uk

Specialty section: This article was submitted to

Exercise Physiology, a section of the journal

Frontiers in Physiology

Received: 16 October 2018 Accepted: 06 December 2018 Published: 08 January 2019

Citation: Lee BJ, Gibson OR, Thake CD, Tipton M, Hawley JA and Cotter JD (2019) Editorial: Cross Adaptation and Cross Tolerance in Human Health and Disease. Front. Physiol. 9:1827. doi: 10.3389/fphys.2018.01827 hypoxia (Lunt et al., 2010), hypoxia to heat (Sotiridis et al., 2018), combined stressors (Takeno et al., 2001; Neal et al., 2017), and more mechanistic (signaling) data from animal models exposed to substantive volumes of stress (Maloyan and Horowitz, 2002, 2005). The role of nutrient availability and the nutrient-exercise interactions which drive phenotypic adaptations to skeletal muscle exposed to a multitude of stressors is also a growing field of interest (Hawley et al., 2018). This research topic includes publications which address both clinical and exercise-centric aspects allied to Cross-adaptation and Cross-tolerance in Human Health and Disease.

An excellent primer covering aspects of preconditioning, short and long term heat acclimation cross-tolerance, and heat acclimation memory in both animal and human experimental models has been provided by Professor Horowitz of the Hebrew University. This extensive review of the cellular and molecular responses underpinning cross-adaptation concludes that the dynamic epigenetic phenomenon not only induces long-lasting cross-tolerance, but enables preservation of its physiological beneficial features in a dormant manner. This conclusion is based on an increasing understanding of epigenetic mechanisms of cross-adaptation and the notion of Heat Acclimation-Mediated Cross-Tolerance vs. Preconditioning-Induced Cross-Tolerance, with a specific focus on the extensive publications from the Horowitz laboratory providing evidence for the role of hypoxia inducible 1-alpha (HIF-1 $\alpha$ ) and heat shock proteins (HSPs) in cytoprotection in the ischemic heart. In short, a rapid, short acclimation stimulus re-establishes the physiological, protected heat acclimation phenotype. The transcriptional machinery, however, is a continuum and as such our knowledge of the epigenetic within-life dynamic mechanisms involved, and their rates of both development and decay, is still in its infancy.

The review by Horowitz sets up three original investigations with a clinical focus. Pollak et al. provide original experimental data that evidences the protective effect of heat acclimatization 


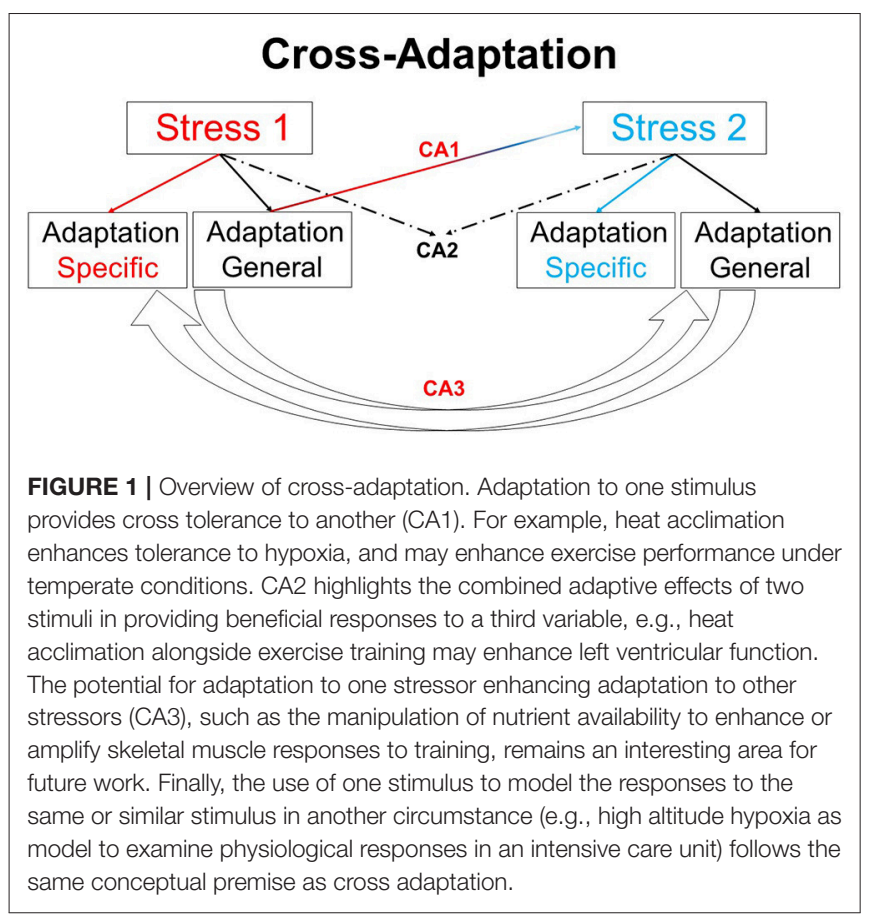

combined with modest exercise training, performed in "free living" conditions, against diastolic dysfunction imposed by ischemic/reperfusion insults in humans. The surgical theme is continued by Barrington et al., who demonstrate the efficacy of using orthopedic perioperative hypoxic air inhalation to prime the local HSP system and attenuate reperfusion stress in a manner similar to that of the current ischemic preconditioning intervention used prior to knee replacement surgery. The benefit of hypoxic air inhalation being that it comes without negative post-surgical side-effects, e.g., delayed wound healing, vascular injury, muscular damage, and greater post-operative pain that can arise as a consequence of ischemia. Hypoxic air inhalation was also the subject of investigation for Morrison et al.; they identify that during 21 days of bed rest, sleep macrostructure is negatively affected, the apnoea-hypopnea index increases, and breathing stability worsens. Each of these metrics become independently exacerbated by continuous exposure to hypoxia. Given the importance of sleep for many clinical and exercise applications where cross-adaptation/tolerance is investigated, interventions using chronic stress such as prolonged hypoxia should be mindful of the potential confounding/maladaptive issues arising from its use.

Exercise related cross-adaptation is studied most extensively within this Research Topic. Leckey et al. present interesting experimental work describing the effects of a 1,3-butanediol acetoacetate (ketone) diester on performance in professional cyclists. In comparison to a placebo, pre-exercise ingestion of the diester results in an impairment in time trial performance that is associated with gut discomfort and higher perception of effort concurrent with elevated serum $\beta$-hydroxybutyrate, serum acetoacetate and urine ketone concentrations. Continuing the exercise and nutrition cross-adaptive theme, papers by
Akerman et al. and Neal et al., investigate the role of fluid balance, and more specifically dehydration as an additional stressor during exercise-heat stress. To understand mechanisms by which manipulating fluid balance may augment adaptation to exercise-heat stress, Akerman et al. prescribed calisthenics in temperate conditions, in hot conditions whilst euhydrated, and in hot conditions with dehydration. Following the three conditions the authors conclude that transient dehydration with heat potentiates short-term (24-h) hematological (hypervolemic) and cardiovascular (hypotensive) outcomes. Neal et al.'s work extends understanding of the relevance of fluid intake during heat acclimation protocols (repeated exercise heat stress) by identifying that when thermal-strain is matched, permissive dehydration which induces a mild, transient, hypohydration does not reliably affect the acquisition or decay of heat acclimation, or endurance performance parameters. Consequently, the current guidance relating to inducing heat adaptation using acclimation remains salient i.e., irrespective of hydration status, trained individuals require $>5$ days to optimize heat acclimation.

Since the emergence of seminal work in the field, heat has been the most widely considered stressor for cross-adaptation. Lee and Thake follow a previous publication in the area (Lee et al., 2016) with important insights into the responses of circulating inflammatory markers during acclimation to control, hypoxic or hot conditions, and subsequent hypoxic stress tests. Whilst heat acclimation induces more immediate and greater changes in monocyte HSP72 in comparison to equivalent training in hypoxia, neither regime attenuated the systemic cytokine response or intestinal damage following acute exercise in hypoxia. This experiment identified that 10 days of fixedwork acclimation does not induce full cytoprotective adaptation, and cellular acclimation homeostasis had yet to be achieved. Further studies are therefore warranted to determine the optimal heat "dose" to maximize the constitutive HSP72 reserves and achieve the potent cross-tolerance effects observed in rodent models (Maloyan and Horowitz, 2002, 2005). Once the optimal dose and associated rates of development and decay are better understood in humans, cross-adaptive interventions aimed at enhancing hypoxic tolerance and operational effectiveness in human occupational, military, and sporting scenarios can be explored. These applied fields can draw upon the data of Tuttle et al. who demonstrate that an acute bout of hot downhill running is an effective preconditioning strategy that ameliorates physiological strain, soreness, and Hsp72 and Hsp90 $\alpha$ mRNA responses of muscle and leukocytes, to a subsequent bout of hot downhill running - these responses being indicative of cross-adaptation. The ability to elicit physiological and cellular protection to exercise heat stress following just a single bout of exercise is appealing for those individuals where extensive periods of time prior to exercise in extreme environments is not possible. For example, cross-adaptation and preconditioning principles could be considered when examining interventions aimed at preparing vulnerable populations, such as the elderly and those with chronic non-communicable diseases, before the onset of debilitating heatwave events that increase morbidity and mortality in these populations (Kenny et al., 2010). For the researcher, these data also valuably demonstrate 
the efficacy of utilizing circulating tissue (leukocytes) in place of muscle samples to infer changes in the heat shock response.

The final inclusion in this research topic brings the collection full circle by considering exercise and clinical cross overs. Sahl et al. investigate how excessive repeated prolonged exercise influence low-grade inflammation and adipose tissue anti-inflammatory macrophage content in six older male recreationally-trained cyclists. The data support the conclusion that regular prolonged exercise does not influence abdominal adipose tissue inflammation, but a higher plasma IL-6 concentration concurrent with a trend toward higher insulin resistance and decreased $\mathrm{VO}_{2 \text { peak }}$, implies that excessive exercise probably attenuates its potential anti-inflammatory effects. The balance between successfully inducing an adaptation and providing too great a stimulus and thereby mitigating the desired response is a delicate balance, and all research

\section{REFERENCES}

Gibson, O. R., Taylor, L., Watt, P. W., and Maxwell, N. S. (2017). Cross-adaptation: heat and cold adaptation to improve physiological and cellular responses to hypoxia. Sports Med. 47, 1751-1768. doi: 10.1007/s40279-017-0717-z

Gibson, O. R., Turner, G., Tuttle, J. A., Taylor, L., Watt, P. W., and Maxwell, N. S. (2015). Heat acclimation attenuates physiological strain and the HSP72, but not $\mathrm{HSP} 90 \alpha$, mRNA response to acute normobaric hypoxia. J. Appl. Physiol. 119, 889-899. doi: 10.1152/japplphysiol.00332.2015

Hawley, J. A., Lundby, C., Cotter, J. D., and Burke, L. M. (2018). Maximizing cellular adaptation to endurance exercise in skeletal muscle. Cell Metab. 27, 962-976. doi: 10.1016/j.cmet.2018.04.014

Heled, Y., Peled, A., Yanovich, R., Shargal, E., Pilz-Burstein, R., Epstein, Y., et al. (2012). Heat acclimation and performance in hypoxic conditions. Aviat. Space Environ. Med. 83, 649-653. doi: 10.3357/ASEM.3241.2012

Kenny, G. P., Yardley, J., Brown, C., Sigal, R. J., and Jay, O. (2010). Heat stress in older individuals and patients with common chronic diseases. CMAJ 182, 1053-1060. doi: 10.1503/cmaj.081050

Lee, B. J., Emery-Sinclair, E. L., Mackenzie, R. W., Hussain, A., Taylor, L., James, R. S., et al. (2014a). The impact of submaximal exercise during heat and/or hypoxia on the cardiovascular and monocyte HSP72 responses to subsequent (post 24 h) exercise in hypoxia. Extreme Physiol. Med. 3:15. doi: 10.1186/20467648-3-15

Lee, B. J., Mackenzie, R. W. A., Cox, V., James, R. S., and Thake, C. D. (2014b). Human monocyte heat shock protein 72 responses to acute hypoxic exercise after 3 days of exercise heat acclimation. Biomed. Res. Int. 72, 1-16. doi: $10.1155 / 2015 / 849809$

Lee, B. J., Miller, A., James, R. S., and Thake, C. D. (2016). Cross acclimation between heat and hypoxia: heat acclimation improves cellular tolerance and exercise performance in acute normobaric hypoxia. Front. Physiol. 7:78. doi: 10.3389/fphys.2016.00078

Lunt, H. C., Barwood, M. J., Corbett, J., and Tipton, M. J. (2010). Cross-adaptation: the effect cold habituation has on the physiological responses to acute hypoxia in humans. J. Physiol. 588, 3605-3613. doi: 10.1113/jphysiol.2010.193458

Maloyan, A., Eli-Berchoer, L., Semenza, G. L., Gerstenblith, G., Stern, M. D., and Horowitz M. (2005). HIF-1 $\alpha$-targeted pathways are activated by heat acclimation and contribute to acclimation-ischemic cross-tolerance in the heart. Physiol. Genomics 23, 79-88. doi: 10.1152/physiolgenomics.00279.2004 into cross-adaptation should be mindful of the challenges of prescribing appropriate exercise intensities, particularly when dealing with individuals of widely varying levels of fitness and when seeking to complement exercise with environmental stress such as heat and hypoxia, or nutritional and dehydration manipulations.

These articles add to our understanding of the whole body, systemic and molecular responses to a multitude of stressors that interact with one another under the cross-adaptation paradigm. The sporting, occupational, and clinical relevance of these areas is only recently being discovered and we look forward to new applied and mechanistic data in this growing area.

\section{AUTHOR CONTRIBUTIONS}

BL and OG: design and scripture. CT, MT, JH and JC: completion and correction.

Maloyan, A., and Horowitz, M. (2002). $\beta$-Adrenergic signaling and thyroid hormones affect HSP72 expression during heat acclimation. J. Appl. Physiol. 93, 107-115. doi: 10.1152/japplphysiol.01122.2001

Neal, R. A., Prout, J., Costello, J., Massey, H. C., Tipton, M. J., Young, J. S., et al. (2017). The effects of 10 days of separate heat and hypoxic exposure on heat acclimation and temperate exercise performance. Am J Physiol. Regul. Integr. Comp. Physiol. 313, R191-R201. doi: 10.1152/ajpregu. 00103.2017

Salgado, R. M., Sheard, A. C., Vaughan, R. A., Parker, D. L., Schneider, S. M., Kenefick, R. W., et al. (2017). Mitochondrial efficiency and exercise economy following heat stress: a potential role of uncoupling protein 3. Physiol. Rep. 5:e13054. doi: 10.14814/phy2.13054

Sotiridis, A., Debevec, T., McDonnell, A. C., Ciuha, U., Eiken, O., and Mekjavic, I. B. (2018). Exercise cardiorespiratory and thermoregulatory responses in normoxic, hypoxic and hot environment following 10-day continuous hypoxic exposure. J. Appl. Physiol. doi: 10.1152/japplphysiol.0111 4.2017. [Epub ahead of print].

Takeno, Y., Kamijo, Y. I., and Nose, H. (2001). Thermoregulatory and aerobic changes after endurance training in a hypobaric hypoxic and warm environment. J. Appl. Physiol. 91, 1520-1528. doi: 10.1152/jappl.2001.91.4.1520

Tipton, M. (2012). A case for combined environmental stressor studies. Extreme Physiol. Med. 1:7. doi: 10.1186/2046-7648-1-7

White, A. C., Salgado, R. M., Astorino, T. A., Loeppky, J. A., Schneider, S. M., McCormick, J. J., et al. (2016). The effect of ten days of heat acclimation on exercise performance in acute hypobaric hypoxia $(4350 \mathrm{~m})$. Temperature 3, 176-185. doi: 10.1080/23328940.2015.1072659

Conflict of Interest Statement: The authors declare that the research was conducted in the absence of any commercial or financial relationships that could be construed as a potential conflict of interest.

Copyright (c) 2019 Lee, Gibson, Thake, Tipton, Hawley and Cotter. This is an openaccess article distributed under the terms of the Creative Commons Attribution License (CC BY). The use, distribution or reproduction in other forums is permitted, provided the original author(s) and the copyright owner(s) are credited and that the original publication in this journal is cited, in accordance with accepted academic practice. No use, distribution or reproduction is permitted which does not comply with these terms. 